\title{
A Case of Probable Diclofenac-Induced Acute Meningism in a Healthy Adolescent
}

\author{
Kai Lun Tang ${ }^{\mathrm{a}, \mathrm{c}}$, Yen Lin Teoh ${ }^{\mathrm{b}}$, Hunainah Khairul Anwar ${ }^{\mathrm{a}}$, Jing Ying Fong ${ }^{\mathrm{a}}$
}

\begin{abstract}
Non-steroidal anti-inflammatory drugs (NSAIDs) and paracetamol are the common analgesics used in children who experience mild to moderate pain. NSAIDs-related case of aseptic meningitis or meningism in healthy adolescent is relatively uncommon. We report a case of an 11-year-old boy with underlying bronchial asthma who had recent intake of three doses of diclofenac $(50 \mathrm{mg})$, presented with signs and symptoms mimicking acute meningism. The boy initially presented to the emergency department after he experienced throbbing headache, dizziness, nausea and persistent vomiting for about 15 times per day for the past 2 days. He did not report presence of any auditory or visual hallucination; however, he claimed to have a bit of photophobia. Signs and symptoms occurred soon after the second dose of diclofenac. Besides, he was also taking oral antibiotic Augmentin for his left epididymo-orchitis problem and famotidine for prevention of gastritis prescribed by general practitioner. Vital signs were normal, and review of systems did not find any abnormality. Neurological assessment found no significant deficit, no cerebellar sign, negative Babinski test and no sign of meningism. Patient did not have frequent fall, head trauma or any family history of neurological diseases. A provisional diagnosis of meningoencephalitis was made after review by specialist. The parents of the boy did not give consent to conduct spinal tap. Upon further examination, urgent contrast-enhanced computed tomography was done and clinical neurological assessment was not suggestive of meningoencephalitis. Antibiotics treatment for meningoencephalitis was stopped $12 \mathrm{~h}$ after hospitalization. Although this case was not diagnosed as aseptic meningitis, timeline of diclofenac intake, onset of symptoms, clinical manifestations and quick resolution symptoms after drug discontinuation were supportive of a temporal relationship between diclofenac and meningism. Paracetamol was given to relieve the headache, vomiting resolved after one dose of intravenous metoclopramide plus ranitidine, and patient was well throughout the 5 days in ward. This adverse effect of NSAIDs is very rare and continuous effort in pharmacovigilance can help to raise awareness among clinicians.
\end{abstract}

Manuscript submitted February 15, 2020, accepted February 21, 2020

aPharmacy Department, Seberang Jaya Hospital (Penang), Ministry of Health, Malaysia

bPediatric Department, Seberang Jaya Hospital (Penang), Ministry of Health, Malaysia

'Corresponding Author: Kai Lun Tang, Pharmacy Department, Seberang Jaya Hospital (Penang), Ministry of Health, Malaysia.

Email: tangkailun@hotmail.com

doi: https://doi.org/10.14740/ijcp358
Keywords: NSAIDs; Acute meningism; Pediatric; Adverse drug reaction; Diclofenac

\section{Introduction}

Non-steroidal anti-inflammatory drugs (NSAIDs) are drugs that have anti-inflammatory, analgesic and antipyretic effects. Although most are not recommended or licensed for children, they have gradually become increasingly important in the management of both acute and chronic pain in children. Ibuprofen and diclofenac are the two frequently used NSAIDs in pediatrics; other NSAIDs such as naproxen are generally used in inflammatory conditions such as juvenile idiopathic arthritis. Gastrointestinal irritation, nephrotoxicity, cutaneous allergy reactions and bronchospasm are among the few adverse reactions that are commonly known to healthcare professionals. However, its adverse effect on central nervous system (CNS) remains inadequately explored. Acute meningitis and meningism associated with NSAIDs use appear to be relatively uncommon $[1,2]$. Cases reported mainly involve patients with underlying autoimmune connective tissue disorder, and ibuprofen is the most frequently implicated culprit for druginduced aseptic meningitis [3-6]. To date, only limited case reports illustrating diclofenac-related CNS adverse effects are available, especially in a healthy adolescent.

\section{Case Report}

A healthy 11-year-old boy, weighing $52 \mathrm{~kg}$, was referred from private health clinic through the emergency department with chief complaint of acute onset of persistent headache, dizziness, nausea and multiple episodes of vomiting at home. Signs and symptoms occurred since past 2 days soon after he started on two doses of diclofenac $50 \mathrm{mg}$ prescribed by a general practitioner for his left epididymo-orchitis problem as pain reliever. Besides, he was also prescribed with oral amoxicillin/ clavulanate and famotidine. He described headache as throbbing in nature, with a pain score of $9 / 10$, and partially relieved after vomiting. He vomited more than 20 episodes on the first day and about 10 - 15 times on the second day, with no specific time, non-projectile, greenish in color. Diclofenac was ingested at 8 am and second dose at $3 \mathrm{pm}$; he started to feel unwell, with reduced appetite, dizzy, nausea, headache and vomited 

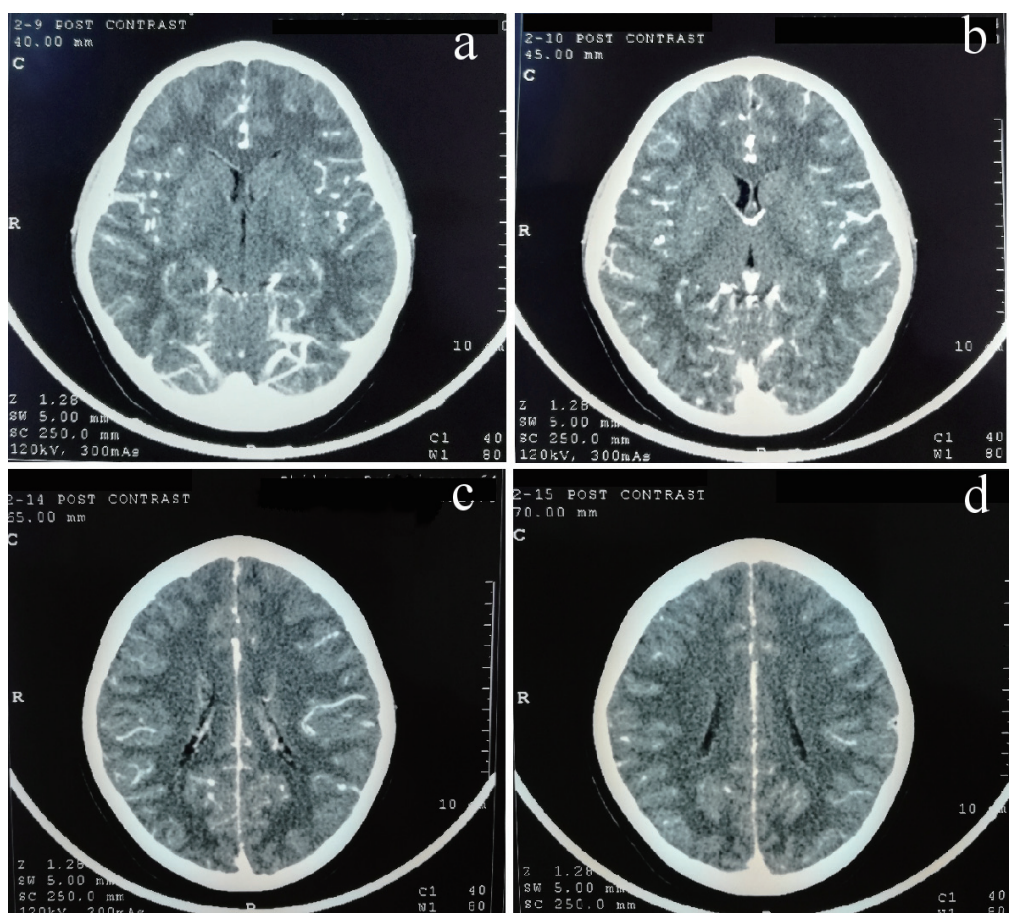

Figure 1. Contrast-enhanced computed tomography (CECT) scan of brain. No focal enhancing brain lesion or abnormal leptomeningeal enhancement, no intracranial bleed.

starting around $6 \mathrm{pm}$. Last dose of diclofenac was ingested at 9 pm before he stopped taking the medication.

According to patient and his parents, he has no history of recent head trauma or fall. Parents claimed that patient did not have abnormal behavior or history of fitting episode since childhood. He has no recent fever at home, no abdominal pain, no issue in passing urine, no dysuria, bowel output is normal and no diarrhea. He did not report presence of any auditory or visual hallucination. However, he claimed to have a bit of photophobia. He is currently in standard 5, and performance is average in school, currently studying in the first class among the three classes. He is active in school, sports and socializes well.

On admission, patient was alert, conscious, orientated but appeared lethargic. Vital signs were normal (blood pressure (BP) $113 / 68 \mathrm{~mm} \mathrm{Hg}$, oxygen saturation $\left(\mathrm{SPO}_{2}\right) 98 \%$, pulse rate (PR) 109 beats/min, respiratory rate (RR) 30 breaths/min, temperature $37.3^{\circ} \mathrm{C}$ ). Physical examinations noted tongue was mildly coated, lethargy looking, fully conscious, equal and reactive pupils $3 \mathrm{~mm} / 3 \mathrm{~mm}$. Review of systems did not find any abnormality and no neurological deficit. Neurological assessment found no cerebellar sign, negative Babinski test and no specific sign of meningitis. An urgent brain contrast-enhanced computed tomography (CECT) was ordered to rule out intracranial bleed and meningoencephalitis. He was given one dose of intravenous hydrocortisone prior to CECT and was kept nil by mouth. Full maintenance intravenous (IV) drip was in place. Venous blood gas (VBG) was taken to rule out acidosis caused by prolonged vomiting and all parameters were within normal limit ( $\mathrm{pH} 7.39$, partial pressure of carbon dioxide $\left(\mathrm{PCO}_{2}\right) 44 \mathrm{~mm}$ $\mathrm{Hg}$, partial pressure of oxygen $\left(\mathrm{PO}_{2}\right) 20 \mathrm{~mm} \mathrm{Hg}$, bicarbonate $\left(\mathrm{cHCO}_{3}^{-}\right) 24 \mathrm{mEq} / \mathrm{L}$, base excess (BE) 1.6). He had a normal white blood cell count of $7.4 \times 10^{9} / \mathrm{L}$, with a normal distribution of neutrophils $5.11 \times 10^{9} / \mathrm{L}(69 \%)$, lymphocytes $1.5 \times 10^{9} / \mathrm{L}$ $(20 \%)$, monocytes $0.76 \times 10^{9} / \mathrm{L}(10 \%)$, basophil $0.02 \times 10^{9} / \mathrm{L}$ $(0.3 \%)$ and eosinophils $0.02 \times 10^{9} / \mathrm{L}(0.3 \%)$. Other cell counts were also normal, i.e. hemoglobin $12.2 \mathrm{~g} / \mathrm{dL}$ and platelet $402 \times$ $10^{9} /$ L. Lumbar puncture was not pursued for cerebrospinal fluid (CSF) analysis due to family refusal for procedure despite clear explanation on importance of investigation. Renal profile (urea $4 \mathrm{mmol} / \mathrm{L}, \mathrm{Na}^{+} 136 \mathrm{mmol} / \mathrm{L}, \mathrm{K}^{+} 3.5 \mathrm{mmol} / \mathrm{L}$, serum creatinine (SrCr) $49 \mu \mathrm{mol} / \mathrm{L}$ ), liver profile (albumin $39 \mathrm{~g} / \mathrm{L}$, alanine aminotransferase (ALT) $18 \mathrm{U} / \mathrm{L}$, alkaline phosphatase (ALP) 216 $\mathrm{U} / \mathrm{L}$ ) and C-reactive protein (CRP) $2.6 \mathrm{mg} / \mathrm{L}$ ) were all within normal limit. Blood culture and sensitivity tested negative of any bacterial growth. Findings from brain CECT (Fig. 1) confirmed no acute intracranial bleed, no focal enhancing lesion or abnormal leptomeningeal enhancement, and no acute hydrocephalus. Timeline between diclofenac use and symptoms onset/resolution of CNS adverse effects were consistent in supporting the relationship. This further strengthens the possibility of diclofenac-induced meningism.

During hospitalization, he was treated empirically with IV ceftriaxone $2 \mathrm{~g}$ 12-hourly and IV acyclovir $720 \mathrm{mg}$ ter die sumendum (TDS). Headache was treated with oral paracetamol $500 \mathrm{mg}$ quater in die (QID), and his condition improved remarkably. Antibiotics were subsequently discontinued $12 \mathrm{~h}$ after admission once meningoencephalitis was ruled out, and diagnosis changed to possible diclofenac-related acute meningism. Rechallenge with offending drug was not done in ward to confirm the diagnosis due to ethical concern. Assessment on the probability of adverse drug reactions was done using Naranjo scale [7], which resulted in a "Probable" relationship 


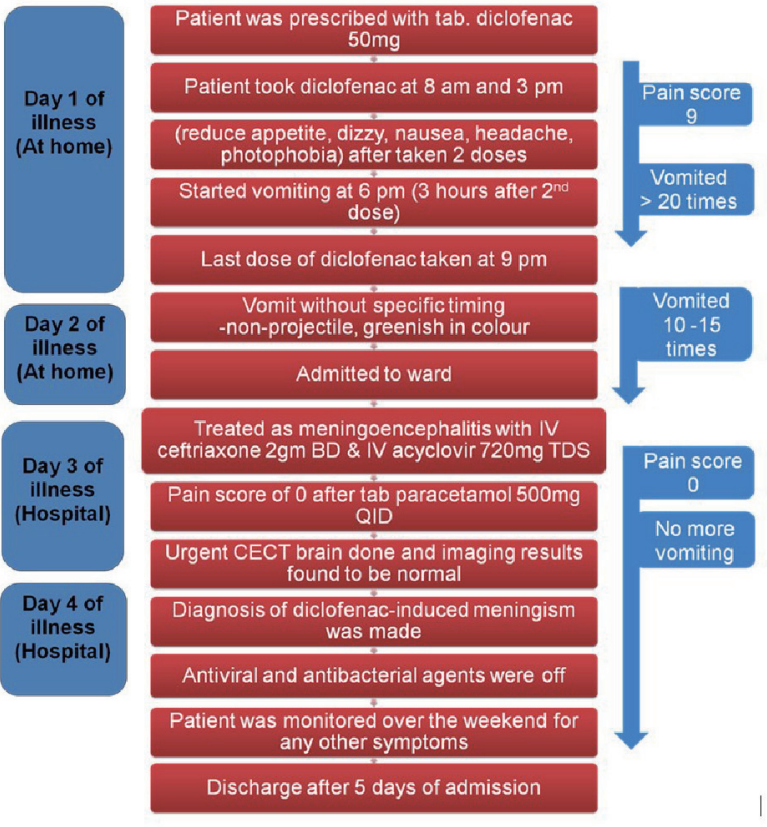

Figure 2. Chronology of events and clinical progress. CECT: contrastenhanced computed tomography.

between acute meningism and intake of diclofenac. The drug adverse reaction causality categorized as "Probable" using WHO-UMC assessment criteria [8] also supported our final diagnosis of probable diclofenac-induced acute meningism. A summary of the chronology of events and patient's clinical progress was shown in Figure 2.

Patient's signs and symptoms in ward resolved completely without any recurrent episodes of vomiting within the 5 days of hospitalization. Patient remained well at 1-month follow-up after discharged. An adverse drug reaction card was provided to patient, and his parents were advised concerning the possible rare adverse effect of diclofenac-related acute meningism whereby future uses of NSAIDs require close supervision by parents and healthcare professionals.

\section{Discussion}

Aseptic meningitis and meningism are both rare adverse effects of NSAIDs. Case reports related to NSAID-induced aseptic meningitis and meningism were published, and majority of the cases involved patients with autoimmune diseases, e.g. rheumatologic disorders, systemic lupus erythematous, and mixed connective tissue disorder $[3,4,6]$. French pharmacovigilance database on drug-induced aseptic meningitis [9] provided healthcare professionals with the list of drugs which were reported to have caused aseptic meningitis and the presenting signs and symptoms. Although many of the cases involved NSAIDs use in patients with underlying immunerelated disorders, this adverse event has also been reported, similar to our case, in previously healthy individuals $[10,11]$.

The pathogenic mechanism of NSAID-induced aseptic meningitis and meningism remain unclear. Some clinicians pro- posed that this adverse effect is consistent with the hypersensitivity [12] and meningeal irritation theory [2,9]. Initially, we had no clue whether it was a case of meningoencephalitis or drug-induced aseptic meningitis. The diagnosis of diclofenacinduced meningism is difficult and usually infectious etiologies need to be excluded. If clinicians missed this early sign of CNS infection, patient may later develop high-spiking temperature and multiple seizures. Hence, broad-spectrum antibiotics were started to cover for meningoencephalitis. This defensive prescribing approach was also seen in other similar cases reported where antibiotics were initially started and later off once diagnosis of drug-induced aseptic meningitis was confirmed $[4,5,11]$.

The initial clinical manifestations of bacterial/viral meningitis and drug-induced aseptic meningitis or meningism were similar. Common manifestations include fever, chills, headache, neck stiffness, nausea, vomiting, and generalised arthralgia/myalgia [2-6]. However, recovery from bacterial/viral meningitis usually takes more than 1 week, some may have neurological sequelae. Following the proposed hypersensitivity and meningeal irritation theory, signs and symptoms of NSAID-induced meningism may become evident a few minutes to several hours after intake of NSAIDs [11, 12]. Most of the cases of druginduced aseptic meningitis or meningism reported quick resolution of signs and symptoms, usually within several hours [11, 13]. Consistent with a few similar case reports [10, 14], our patient's signs and symptoms mimicking meningitis/meningism became evident several hours after intake of diclofenac (two doses), and resolved after $24 \mathrm{~h}$ of stopping the offending drug. This quick resolution of signs and symptoms also helped to distinguish drug-induced acute meningism from meningoencephalitis. We did not re-challenge our patient with diclofenac in view of rapid resolution of his signs and symptoms after stopping diclofenac and also due to ethical considerations.

After the patient was subjected to series of tests to screen for infection and brain trauma, the diagnosis of diclofenacinduced acute meningism was made by establishing a temporal relationship with the administration of the drug, onset of clinical symptoms, and rapid resolution after withdrawal. We were unable to provide the CSF analysis findings because parents refused lumbar puncture prior to initiation of antibiotics. Antibiotics treatment were later stopped after $24 \mathrm{~h}$ due to a normal brain CECT findings, rapid recovery and absence of classic signs of meningitis on physical examination, i.e. nuchal rigidity, Kernig's and Brudzinski's signs. Our patients did not present with confusion, and no up-going plantar, hence meningoencephalitis is unlikely. Upon follow-up, we found no cognitive or neurological sequelae reported by clinician who had seen the patient in clinic. Although this case was not diagnosed as aseptic meningitis, timeline of diclofenac intake, onset of symptoms, clinical manifestations and quick resolution symptoms after drug discontinuation were supportive of a temporal relationship between diclofenac and meningism.

Our patient was treated with paracetamol in ward but did not have any similar adverse reactions towards the drug. We advised the parents and the boy to be cautious with NSAIDs use, and if possible, to avoid usage of diclofenac and ibuprofen. In current clinical practice, NSAID-induced aseptic meningitis/meningism remained a diagnosis of exclusion. There is no direct method to confirm a drug-induced adverse reaction 
except for drug re-challenge test, which involves ethical concern.

\section{Conclusions}

The clinical course of diclofenac-induced meningism was relatively short and benign, with rapid resolution of symptoms after discontinuing the drug. Recognition of rare NSAIDs-related aseptic meningitis/meningism requires considerable expertise in assessment of clinical manifestations, drug use history and making differential diagnosis. Clinician or pharmacist should be aware of this rare adverse effect of NSAIDs so that accurate diagnosis can be made earlier.

\section{Acknowledgments}

We would like to thank the Director General of Health Malaysia for his permission to publish this article.

\section{Financial Disclosure}

None to declare.

\section{Conflict of Interest}

The authors declare that they have no conflict of interest.

\section{Informed Consent}

Written consent was obtained from patient's caregiver (mother).

\section{Author Contributions}

Kai Lun Tang and Hunainah Khairul Anwar drafted the initial case report, reviewed and revised the manuscript. Hunainah Khairul Anwar registered the case report on National Medical Research Registry (NMRR). Yen Lin Teoh and Jing Ying Fong counter-checked and critically reviewed all clinical and laboratory data and discussions. All authors approved the final manuscript as submitted and agree to be accountable for all aspects of the work.

\section{Data Availability}

Authors declare that data supporting the findings of this study are available within the article.

\section{References}

1. Yelehe-Okouma M, Czmil-Garon J, Pape E, Petitpain N, Gillet P. Drug-induced aseptic meningitis: a mini-review. Fundam Clin Pharmacol. 2018;32(3):252-260.

2. Moris G, Garcia-Monco JC. The challenge of drug-induced aseptic meningitis. Arch Intern Med. 1999;159(11):11851194.

3. Nguyen HT, Juurlink DN. Recurrent ibuprofen-induced aseptic meningitis. Ann Pharmacother. 2004;38(3):408410.

4. Karmacharya P, Mainali NR, Aryal MR, Lloyd B. Recurrent case of ibuprofen-induced aseptic meningitis in mixed connective tissue disease. BMJ Case Rep. 2013;2013:bcr-2013-009571.

5. Lee RZ, Hardiman O, O'Connell PG. Ibuprofen-induced aseptic meningoencephalitis. Rheumatology (Oxford). 2002;41(3):353-355.

6. Rodriguez SC, Olguin AM, Miralles CP, Viladrich PF. Characteristics of meningitis caused by Ibuprofen: report of 2 cases with recurrent episodes and review of the literature. Medicine (Baltimore). 2006;85(4):214-220.

7. Naranjo CA, Busto U, Sellers EM, Sandor P, Ruiz I, Roberts EA, Janecek E, et al. A method for estimating the probability of adverse drug reactions. Clin Pharmacol Ther. 1981;30(2):239-245.

8. The use of the WHO-UMC system for standardised case causality assessment. Accessed from: http:/www.WHOUMC.org/graphics/4409.pdf [last accessed on 25 May 2019].

9. Bihan K, Weiss N, Théophile H, Funck-Brentano C, Lebrun-Vignes B. Drug-induced aseptic meningitis: 329 cases from the French pharmacovigilance database analysis. Br J Clin Pharmacol. 2019; 85: 2540-2546.

10. Lawson JM, Grady MJ. Ibuprofen-induced aseptic meningitis in a previously healthy patient. West J Med. 1985;143(3):386-387.

11. Chazan B, Weiss A, Weiner Z, Rimbrot S, Raz R. Drug induced aseptic meningitis due to diclofenac. J Neurol. 2003;250(12):1503-1504.

12. Moreno-Ancillo A, Gil-Adrados AC, Jurado-Palomo J. Ibuprofen-induced aseptic meningoencephalitis confirmed by drug challenge. J Investig Allergol Clin Immunol. 2011;21(6):484-487.

13. Chaudhry HJ, Cunha BA. Drug-induced aseptic meningitis. Diagnosis leads to quick resolution. Postgrad Med. 1991;90(7):65-70.

14. Codding C, Targoff IN, McCarty GA. Aseptic meningitis in association with diclofenac treatment in a patient with systemic lupus erythematosus. Arthritis Rheum. 1991;34(10):1340-1341. 